\title{
Synthetic Study towards Entecavir
}

\section{Shuangjie Lin ${ }^{1}$, Feifei Huang ${ }^{1}$, Shenkun Yang ${ }^{1}$, Shuangping Huang ${ }^{1,}$, Xiaoji Wang ${ }^{1, b}$}

${ }^{1}$ School of Pharmacy, Jiangxi Science and Technology Normal University, Jiangxi 330013, China a185544590@qq.com, b13767101659@163.com

Keywords: Entecavir; synthesis; HBV

\begin{abstract}
A synthetic design of Entecavir was described, and the intermediate, (S)-N-methoxy -3-((4-methoxybenzyl)oxy)-N-methylpent-4-enamide, was synthesized using acrylaldehyde as the starting material, which was obtained via an Evans Aldol reaction, Weinreb amide formation, and a $\mathrm{PMB}$ protection.
\end{abstract}

\section{Introduction}

Hepatitis B virus (HBV) represents one of the most prevalent viral diseases in the world ${ }^{[1]}$ and is known to be a cause of serious liver disorders. It is reported that more than 400 million people have been chronically infected and there is a continuing need for new therapies for individuals infected with HBV. Entecavir (BMS-200475, 1) (Fig. 1) is a carbocyclic guanosine nucleoside analog with potent selectivity against hepatitis B virus ${ }^{[2]}$ which was approved under the trade name Baraclude in March 2005 by the US Food and Drug Administration for the treatment of chronic HBV infection in adults. Entecavir inhibits DNA synthesis in HBV infected cells in three steps: the priming of the polymerase, the reverse transcription of the pregenomic messenger RNA and the synthesis of the positive strand of HBV DNA. Entecavir is also effected to lamivudine- and adefovir-resistant HBV strains $^{[3]}$.<smiles>C=C1[C@H](CO)[C@@H](O)C[C@H]1n1cnc2c(=O)[nH]c(N)nc21</smiles>

Figure 1. The structure of Entecavir

As Entecavir's remarkable potency, resistance, and safety profile, a great deal of synthetic effort $^{[4]}$ has been devoted to the enantiospecific syntheses of Entecavir, among which most reported syntheses employ five-membered carbocyclic compounds as the starting material. In our study, we would describe our synthetic analysis towards Entecavir using acrylaldehyde as the starting material to construct the protected five member ring Entecavir $\mathbf{1}$ and our present work of the synthesis towards Entecavir. Our retrosynthetic route is outlined in Scheme 2. 


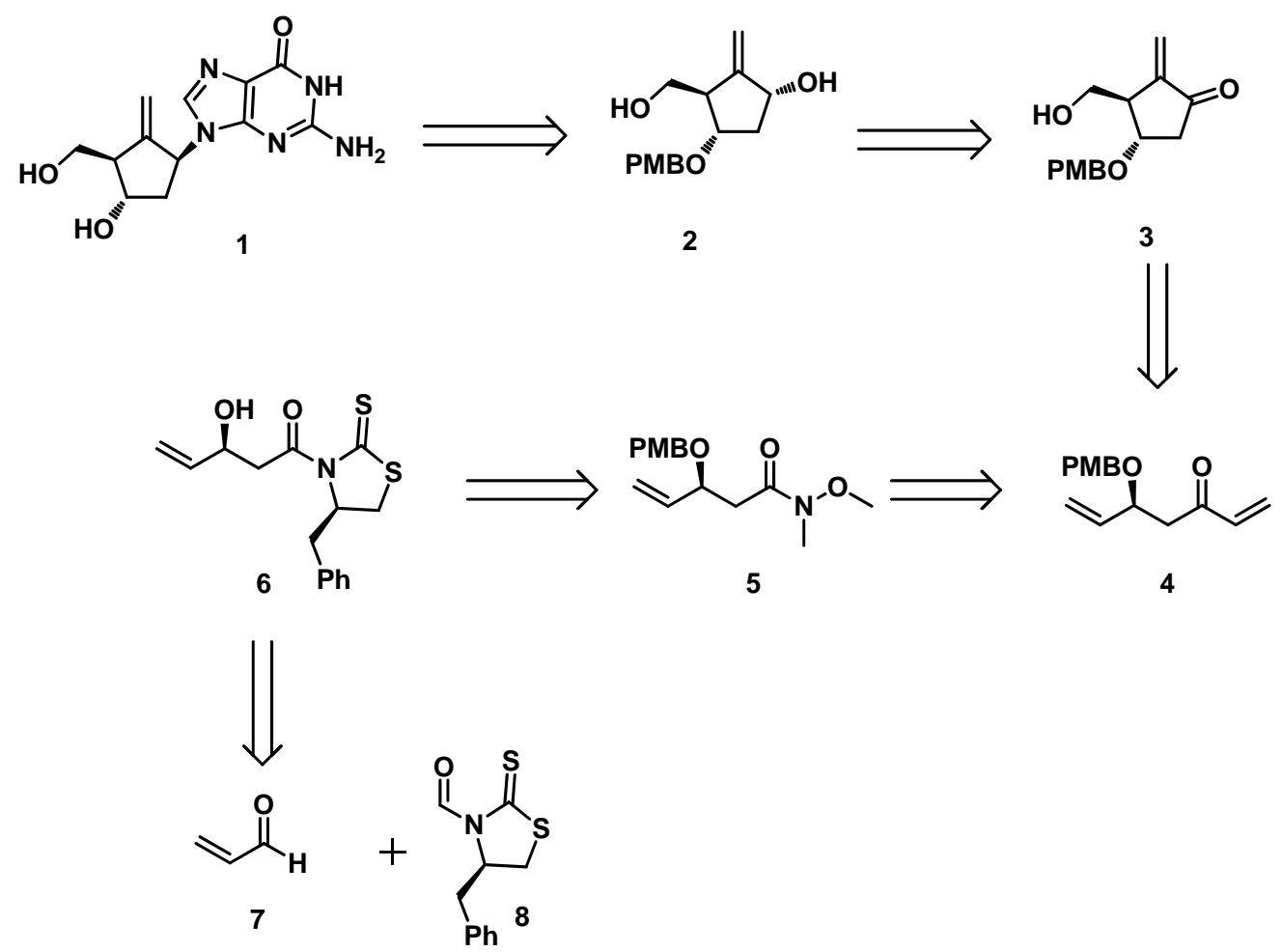

Scheme 2. Our retrosynthetic analysis of Entecavir

As shown above, for the stereoselective introduction of the purine base, we planned to perform a Mitsunobu reaction between a substituted purine and allylic hydroxyl group of compound 2, which could be easily obtained after a diastereoselective reduction from ketone $\mathbf{3}$. The intermediate $\mathbf{3}$ was designed to be got from alkene $\mathbf{4}$, and compound $\mathbf{4}$ could in turn be got from amide $\mathbf{5}$ via a Weinreb reaction with a Grignard reagent. The amide $\mathbf{5}$ could be smoothly obtained from compound $\mathbf{6}$ via a nucleophilic substitution. For the compound 6, it would be readily got through an Evans Aldol reaction between acrylaldehyde 7 and Evans chiral auxiliary 8. In this paper, we just reported the synthesis of intermediate 5 . The detailed route of compound 5 was shown in Scheme 3.

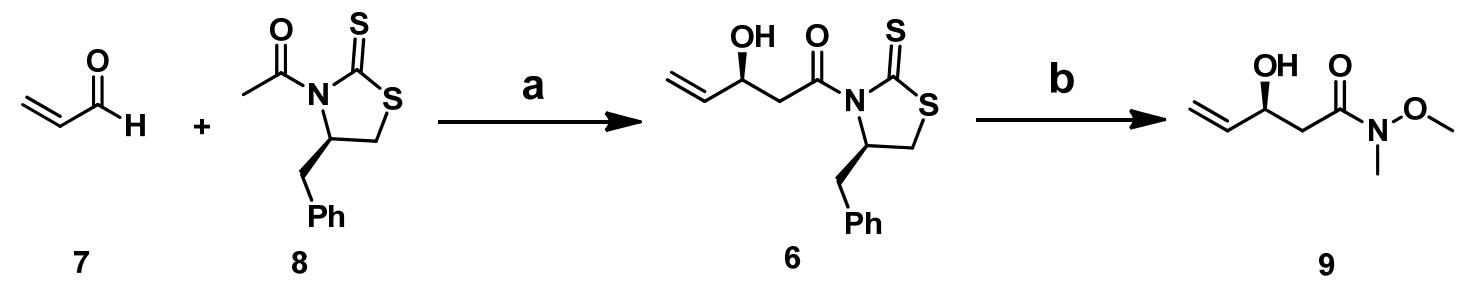<smiles>C=C(CO)C1CC(O)C(C)C1n1cnc2c(=O)[nH]c(N)nc21</smiles>

a) $\mathrm{TiCl}_{4}$, DIPEA, DCM, $0^{\circ} \mathrm{C}$ to $-78^{\circ} \mathrm{C}, 2 \mathrm{~h} ;$ b) $\mathrm{MeONHMe} \cdot \mathrm{HCl}$, imidazole, $\mathrm{CH}_{2} \mathrm{Cl}_{2}$, rt, overnight; c) $\mathrm{NaH}, \mathrm{PMBBr}, \mathrm{DMF},-10^{\circ} \mathrm{C}, 30 \mathrm{~min}$

Scheme 3. Synthetic route of compound 5

\section{Experimental}

NMR spectra were recorded on Bruker AV-400MHz spectrometers. The solvents and reagents were purified and dried according to standard procedures: $\mathrm{CH}_{2} \mathrm{Cl}_{2}$, THF, DMF were distilled from $\mathrm{CaH}_{2}$ prior to use. 
(S)-1-((R)-4-benzyl-2-thioxothiazolidin-3-yl)-3-hydroxypent-4-en-1-one 6

(R)-1-(4-benzyl-2-thioxothiazolidin-3-yl)ethanone 8 (3.5 g, $14.08 \mathrm{mmol})$ was dissolved in $\mathrm{CH}_{2} \mathrm{Cl}_{2}(72 \mathrm{~mL})$ and was cooled to $0{ }^{\circ} \mathrm{C}$. Titanium tetrachloride $(1.68 \mathrm{ml})$ was added dropwise. The thick suspension was stirred for 10 minutes, upon which diisopropylethylamine $(2.68 \mathrm{~mL})$ was added dropwise at $0{ }^{\circ} \mathrm{C}$. After stirring for $10 \mathrm{~min}$, the solution was cooled to $-78^{\circ} \mathrm{C}$ and to the reaction mixture was added the solution of acrylaldehyde $7(0.85 \mathrm{~mL}, 12.8 \mathrm{mmol})$ in $\mathrm{CH}_{2} \mathrm{Cl}_{2}(22$ $\mathrm{mL})$. The reaction was stirred for another $1 \mathrm{~h}$ and was then quenched with saturated ammonium chloride solution. The solution was extracted with $\mathrm{CH}_{2} \mathrm{Cl}_{2}$, dried over anhydrous $\mathrm{Na}_{2} \mathrm{SO}_{4}$, filtered and concentrated. The residue was purified by flash column chromatography to provide the product $6(3.1 \mathrm{~g}, 79 \%) .{ }^{1} \mathrm{H}$ NMR $\left(400 \mathrm{MHz}, \mathrm{CDCl}_{3}\right): \delta 7.41-7.24(\mathrm{~m}, 5 \mathrm{H}), 5.95$ (ddd, $J=17.3,10.5,5.2 \mathrm{~Hz}$, $1 \mathrm{H}), 5.44-5.30(\mathrm{~m}, 2 \mathrm{H}), 5.17(\mathrm{dt}, J=10.5,1.4 \mathrm{~Hz}, 1 \mathrm{H}), 4.72-4.66(\mathrm{~m}, 1 \mathrm{H}), 3.66(\mathrm{dd}, J=17.3,3.0$ $\mathrm{Hz}, 1 \mathrm{H}), 3.41(\mathrm{dd}, J=12.0,7.5 \mathrm{~Hz}, 1 \mathrm{H}), 3.32(\mathrm{dd}, J=17.3,8.6 \mathrm{~Hz}, 1 \mathrm{H}), 3.23(\mathrm{dd}, J=13.2,3.7 \mathrm{~Hz}$, $1 \mathrm{H}), 3.05$ (dd, $J=13.2,10.5 \mathrm{~Hz}, 1 \mathrm{H}), 2.90$ (d, $J=12.0 \mathrm{~Hz}, 1 \mathrm{H}), 2.88-2.77$ (br s, $-\mathrm{OH}, 1 \mathrm{H})$.

\section{(S)-3-hydroxy-N-methoxy-N-methylpent-4-enamide 9}

To a soltion of alcohol 6 (781 $\mathrm{mg}, 2.54 \mathrm{mmol})$ in $\mathrm{CH}_{2} \mathrm{Cl}_{2}(12.7 \mathrm{~mL})$ were added $\mathrm{MeONHMe} \cdot \mathrm{HCl}(496 \mathrm{mg}, 5.08 \mathrm{mmol})$ and imidazole $(865 \mathrm{mg}, 12.7 \mathrm{mmol})$, and the resultant mixture was stirred at room temperature overnight. The reaction was then quenched with saturated aqueous $\mathrm{NH}_{4} \mathrm{Cl}$ solution, and the resultant mixture was extracted with DCM. The organic layer was washed with brine, dried over anhydrous $\mathrm{Na}_{2} \mathrm{SO}_{4}$, filtered and concentrated under reduced pressure. Purification of the residue by flash chromatography gave Weinreb amide $9(363.7 \mathrm{mg}, 90 \%)$ as a colorless oil. ${ }^{1} \mathrm{H}$ NMR $\left(400 \mathrm{MHz}, \mathrm{CDCl}_{3}\right): \delta 5.92(\mathrm{ddd}, J=17.3,10.5,5.5 \mathrm{~Hz}, 1 \mathrm{H}), 5.32(\mathrm{dt}, J=$ 17.3, $1.4 \mathrm{~Hz}, 1 \mathrm{H}), 5.16$ (dt, $J=10.5,1.4 \mathrm{~Hz}, 1 \mathrm{H}), 4.60-4.54(\mathrm{~m}, 1 \mathrm{H}), 3.79$ (br s, $-\mathrm{OH}, 1 \mathrm{H}), 3.69$ (s, $3 \mathrm{H}), 3.20(\mathrm{~s}, 3 \mathrm{H}), 2.72(\mathrm{~d}, J=16.1 \mathrm{~Hz}, 1 \mathrm{H}), 2.60(\mathrm{dd}, J=16.1,9.1 \mathrm{~Hz}, 1 \mathrm{H})$.

\section{(S)-N-methoxy-3-((4-methoxybenzyl)oxy)-N-methylpent-4-enamide 5}

Under a nitrogen atmosphere, to a solution of compound $9(282 \mathrm{mg}, 1.77 \mathrm{mmol})$ in anhydrous DMF $(5.5 \mathrm{~mL})$ was added $\mathrm{NaH}\left(120 \mathrm{mg}\right.$ of $60 \%$ wt suspension, $3.0 \mathrm{mmol}$ ) at $-10^{\circ} \mathrm{C}$. Then, 1-(bromomethyl)-4-methoxybenzene $(0.45 \mathrm{~mL}, 3.0 \mathrm{mmol})$ was added dropwise. The suspension was stirred at the same temperature for 30 minutes then poured into $\mathrm{H}_{2} \mathrm{O}(3 \mathrm{~mL})$ and pentane/Ether (6 mL each). The layers were separated and the organic layer was sequentially washed with $\mathrm{H}_{2} \mathrm{O}$ and brine. Then it was dried over $\mathrm{Na}_{2} \mathrm{SO}_{4}$ and concentrated. The residue was purified by flash chromatography to provide PMB ether $5(296 \mathrm{mg}, 60 \%)$ as a colorless oil. ${ }^{1} \mathrm{H}$ NMR (400 MHz, $\left.\mathrm{CDCl}_{3}\right): \delta 7.25(\mathrm{~d}, J=8.4 \mathrm{~Hz}, 2 \mathrm{H}), 6.86(\mathrm{~d}, J=8.4 \mathrm{~Hz}, 2 \mathrm{H}), 6.86-5.78(\mathrm{~m}, 1 \mathrm{H}), 5.33(\mathrm{dt}, J=17.3$, $1.4 \mathrm{~Hz}, 1 \mathrm{H}), 5.25(\mathrm{dt}, J=10.5,1.4 \mathrm{~Hz}, 1 \mathrm{H}), 4.51(\mathrm{~d}, J=10.8 \mathrm{~Hz}, 1 \mathrm{H}), 4.39-4.34(\mathrm{~m}, 2 \mathrm{H}), 3.79 \quad$ (s, $3 \mathrm{H}), 3.65(\mathrm{~s}, 3 \mathrm{H}), 3.19(\mathrm{~s}, 3 \mathrm{H}), 2.95-2.89(\mathrm{~m}, 1 \mathrm{H}), 2.57-2.51(\mathrm{~m}, 1 \mathrm{H})$

\section{Acknowledgement}

We thank Science and Technology Planning Project of Jiangxi Province (20142BBE50006), the Science and Technology Project of Jiangxi Province Education Department (GJJ14579), Scientific Research Fund of Jiangxi Provincial Education Department (No.KJLD12036).

\section{References}

[1] Regev, A.; Schiff, E. R. Adv. Inter. Med. 2001, 46, 107.

[2] a) Innaimo, S. F.; Sieffer, M.; Bisacchi, G. S.; Standring, N.; Zahler, R.; Colonno, R. J. Antimicrob. Agents Chemother. 1997, 41, 1444; b) Lai, C.; Rosmawati, M.; Lao, J.; Vlierberghe, H.; Anderson, F.; Thomas, N.; De Hertogh, D. J. Hepatology 2001, 34, 24; c) Bisacchi, G. S.; Chao, S. T.; Bachand, C.; Daris, J. P.; Innaimo, S. F.; Jacobs, G. A.; Kocy, O.; Lapointe, P.; Martel, A.; Merchant, Z.; Slusarchyk, W. A.; Sundeen, J. E.; Young, M. G.; Colonno, R. J.; Zahler, R. Bioorg. Med. Chem. Lett. 1997, 7, 127.

[3] a) Kwak, M. S.; Choi, J. W.; Lee, J. S.; Kim, K. A.; Suh, J. H.; Cho, Y. S.; Won, S. Y.; Park, B. K.; Lee, C. K. J. Viral Hepat. 2011, 18, 432; b) Chang, T. T.; Lai, C. L.; Yoon, S. K.; Lee, S. S.; 
Coelho, H. S. M.; Carrilho, F. J.; Poordad, F.; Halota, W.; Horsmans, Y.; Tsai, N.; Zhang, H.; Tenney, D. J.; Tamez, R.; Iloeje, U. Hepatology. 2010, 51, 422.

[4] a) Ziegler, F. E.; Sarpong, M. A. Tetrahedron 2003, 59, 9013; b) Zhou, B.; Li, Y. C. Tetrahedron Lett. 2012, 53, 502; c) Bisacchi, G. S.; Chao, S. T.; Bachard, C.; Daris, J. P.; Innaimo, S.; Jacobs, G. A.; Kocy, O.; Lapointe, P.; Martel, A.; Merchant, Z.; Slusarchyk, W. A.; Sundeen, J. E.; Young, M. G.; Colonno, R.; Zahler, R. Bioorg. Med. Chem. Lett. 1997, 7, 127; d) Bisacchi, G. S.; Sundeen, J. E. W.O. Patent 9809964, 1998.; e) Pendri, Y. R.; Chen, C. P. H.; Patel, S. S. et al. U. S. Patent 0192912, 2004.; f) Guo, L. W.; Xiao, Y. J.; Yang, L. P. Chin. Chem. Lett. 2006, 17, 907; g) Zhou, M. X.; Reiff, E. A. et al. U. S. Patent 7786300, 2010. 